\title{
KEAP1 knockdown in melanocytes induces cell proliferation and survival via HO-1-associated $\beta$-catenin signaling
}

\author{
Ji Young Kim ${ }^{1}$, Hemin Lee', Eun Jung Lee', Mikyoung Kim ${ }^{2}$, \\ Tae-Gyun Kim ${ }^{2,3}$, Hyoung-Pyo Kim ${ }^{2,3}$, Gehoon Chung ${ }^{4}$, and Sang Ho Oh${ }^{1}$
}

${ }^{1}$ Department of Dermatology and Cutaneous Biology Research Institute, Severance Hospital, Yonsei University College of Medicine, Seoul, Korea, ${ }^{2}$ Department of Environmental Medical Biology, Institute of Tropical Medicine, Yonsei University College of Medicine, Seoul, Korea, ${ }^{3}$ Brain Korea 21 Project for Medical Science, Yonsei University College of Medicine, Seoul, Korea,

${ }^{4}$ Department of Oral Physiology, School of Dentistry, Seoul National University, Seoul, Korea

\section{Figure abstract}

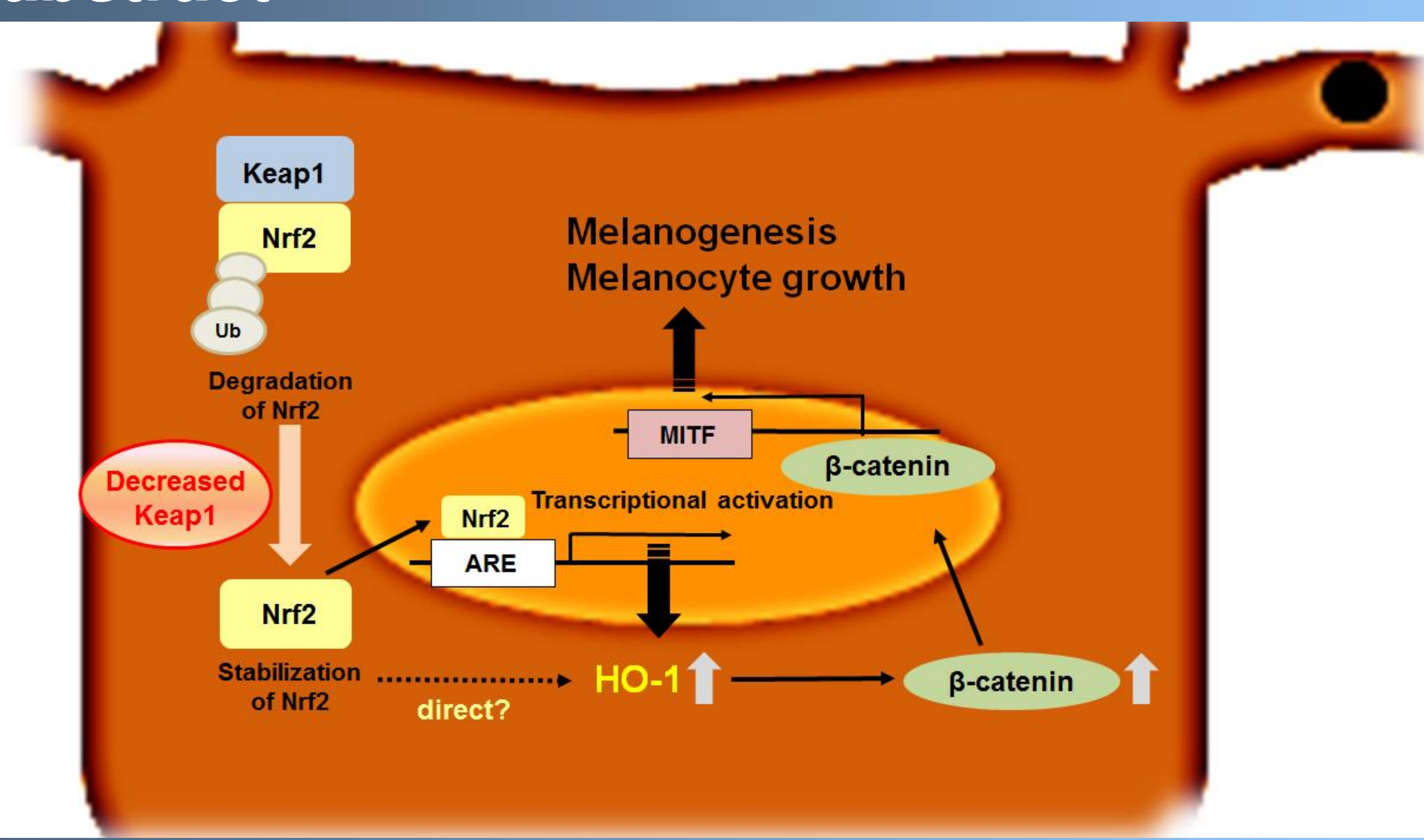

\section{Introduction}

Nrf2-Keap1 signaling pathway

$>$ Plays important roles in protecting cells against oxidative stresses and maintaining skin homeostasis

$>$ HO-1, which is the main target gene of the Nrf2 protects melanocytes against oxidative stress

\section{HO-1 and skin disorders}

$>$ Overexpression of $\mathrm{HO}-1$ has been linked to proliferation of melanoma cells and their resistance to oxidative stress

> Impaired activation of Nrf2 under oxidative stress along with decreased HO1 expression were closely implicated in death of vitiligo melanocytes

Nrf2-Keap1 \& HO-1 and melanogensis

$>$ Still controversial depending on studies

$>$ Investigations directed toward Keap1 functions in melanocytes are limited.

$>$ Therefore, this study aimed to explore how Keap1-silencing affects melanocyte proliferation and survival as well as melanogenesis.

\section{Methods}

Cells: Melan-a (a highly pigmented non-tumorigenic mouse melanocyte cell

line) and primary human epidermal melanocytes (PHEMs)

$>$ Short interfering RNA-targeted gene silencing

$>$ RNA-sequencing analysis

$>$ Real-time RT-PCR, Western blot analysis

$>$ Tyrosinase activity assay \& Measurement of melanin content

\section{Results}

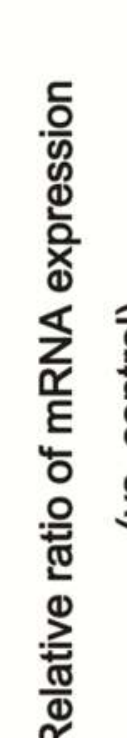
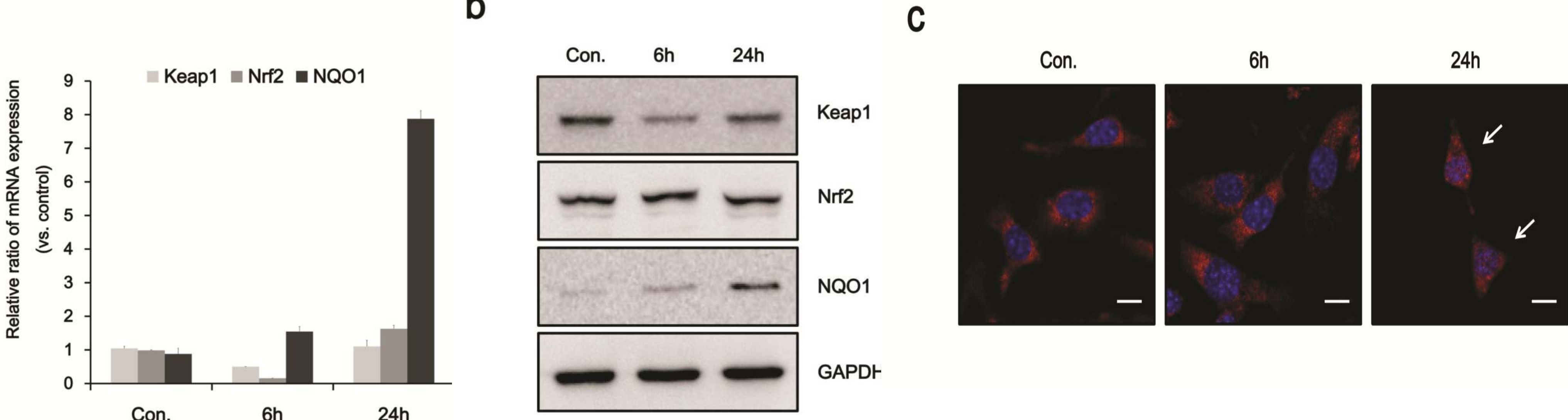

-Fig. 1. Effect of UVB irradiation on Nrf2 signaling. Melan-a cells were irradiated by UVB (20 $\mathrm{mJ} / \mathrm{cm}^{2}$ ). After 6 or $24 \mathrm{~h}$, the cells were harvested, and expression of Keap1, Nrf2 and NQO1 was analyzed by (a) real-time RT-PCR and (b) western blot analysis. (c) The localization of Nrf2 (white arrows) was analyzed using confocal microscopy.
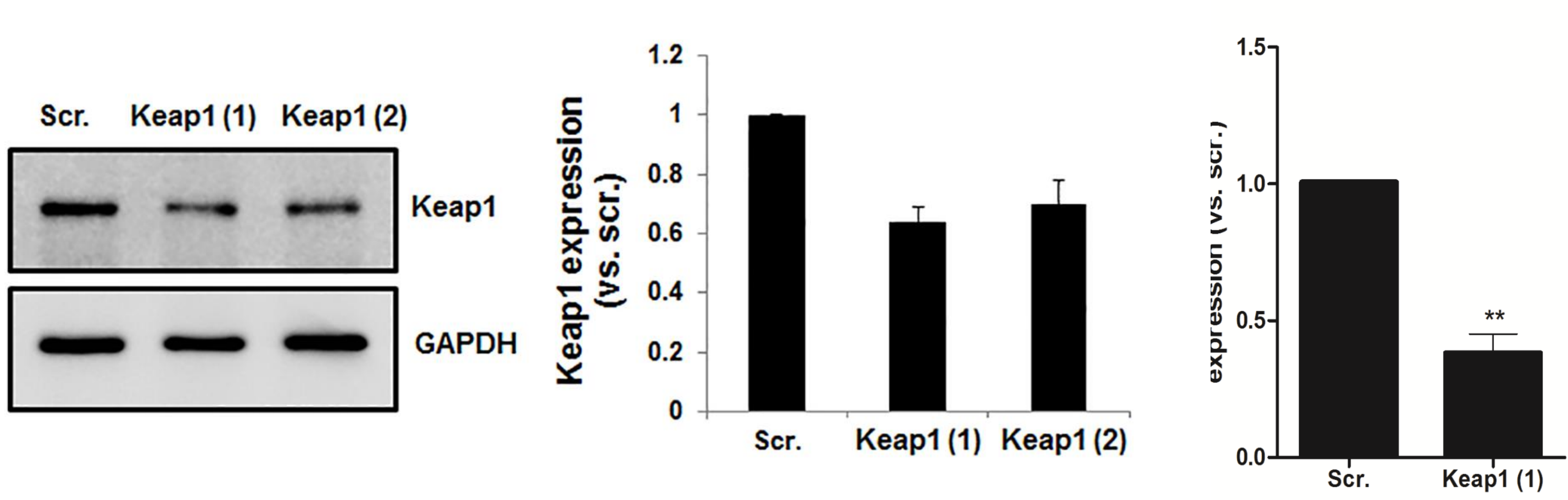

-Fig. 2. Confirmation of Keap1 knockdown in PHEMs using (a) western blotting and (b) realtime RT-PCR
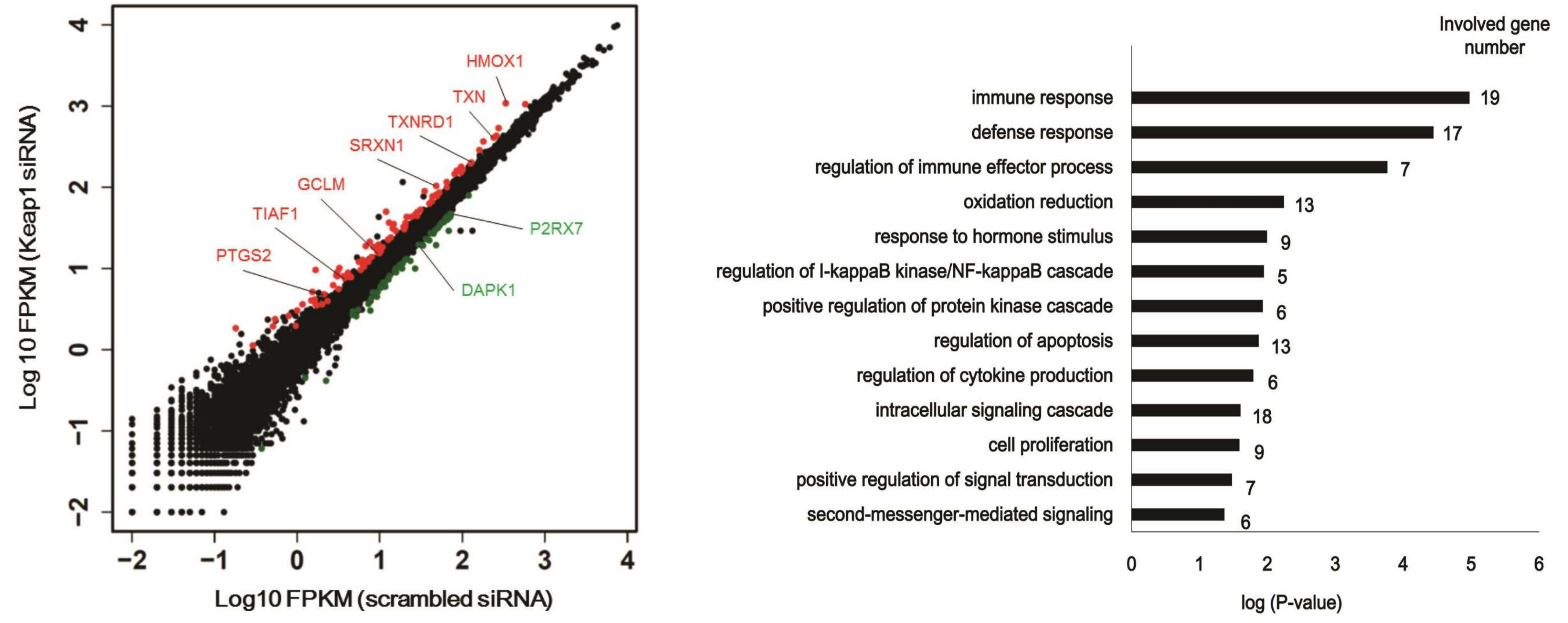

Fig. 3. Gene expression profiling following Keap1 siRNA knockdown in primary human epidermal melanocytes using RNA-seq. (left) Scatter plot of differential gene expression $(\geq 1.5$-fold; $P<0.05)$ in Keap1 siRNA-treated cells versus scrambled siRNA-treated cells, including significantly up-regulated genes (red) and down-regulated genes (green). (right) Summary of selected categories of GO (Gene Ontology) terms as calculated by DAVID.
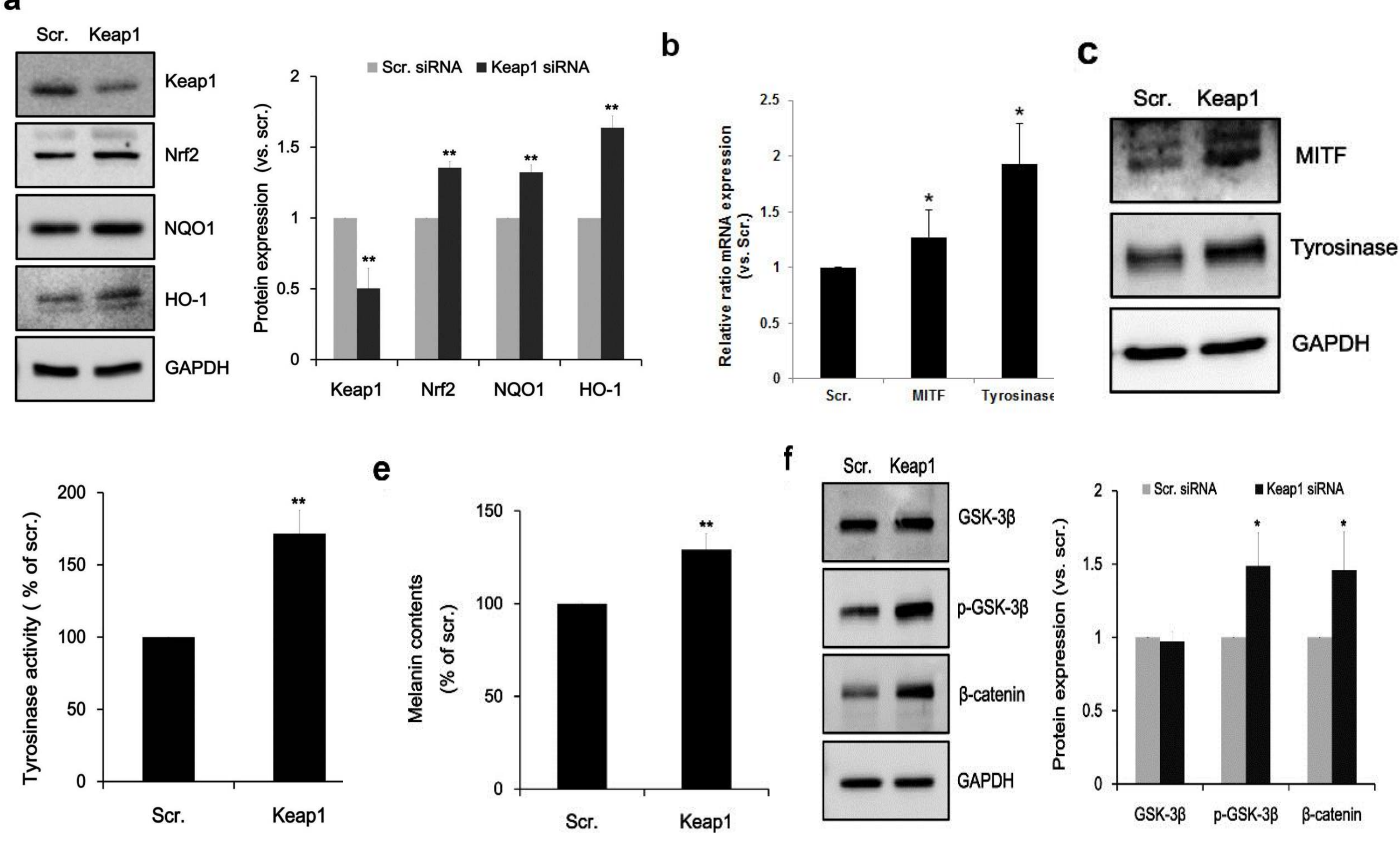

Fig. 4. Effect of Keap1 downregulation on Nrf2 activation and melanogenesis in primary human epidermal melanocytes. The protein expression of Nrf2, NQO1, and HO1 were analyzed by (a) western blot analysis. Expression of MITF and tyrosinase was examined by real-time RT-PCR (48 hr) (b) and western blot analyses (72hr) (c), and the tyrosinase activity (72h) (d) and melanin content (96h) (e) were measured. (f) The expression of GSK-3 $\beta$, p-GSK3 $\beta$-Ser9, and $\beta$-catenin (48h) were investigated.
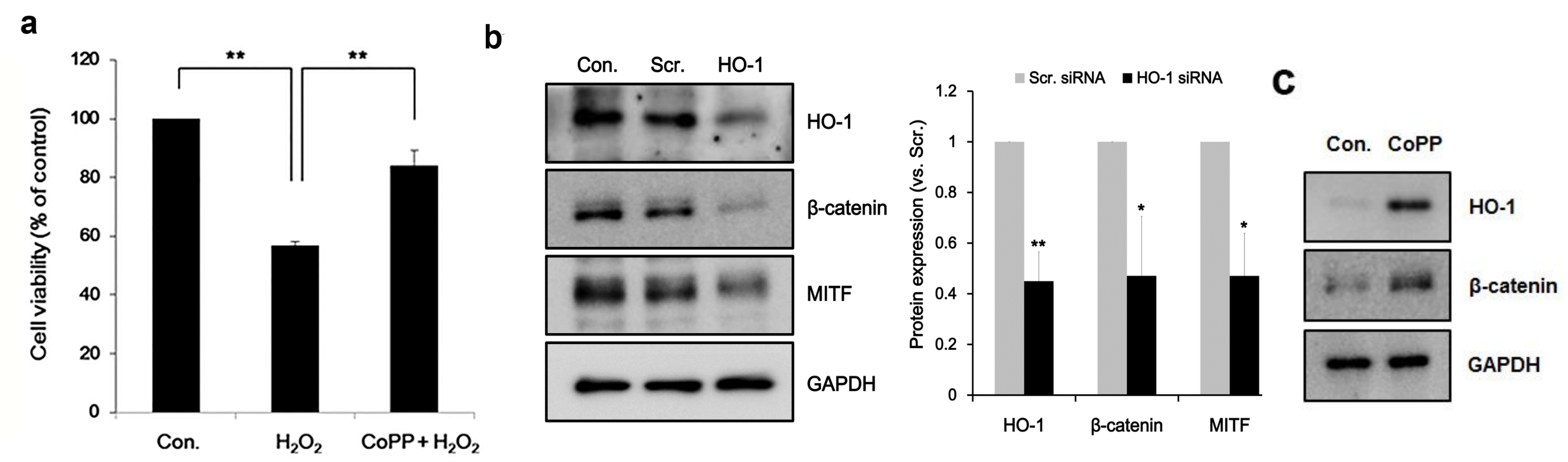

Fig. 5. The effect of HO-1 by CoPP (a HO-1 inducer) on $\mathrm{H}_{2} \mathrm{O}_{2}$-induced cell death, expression levels of MITF and $\beta$-catenin and melanin production a

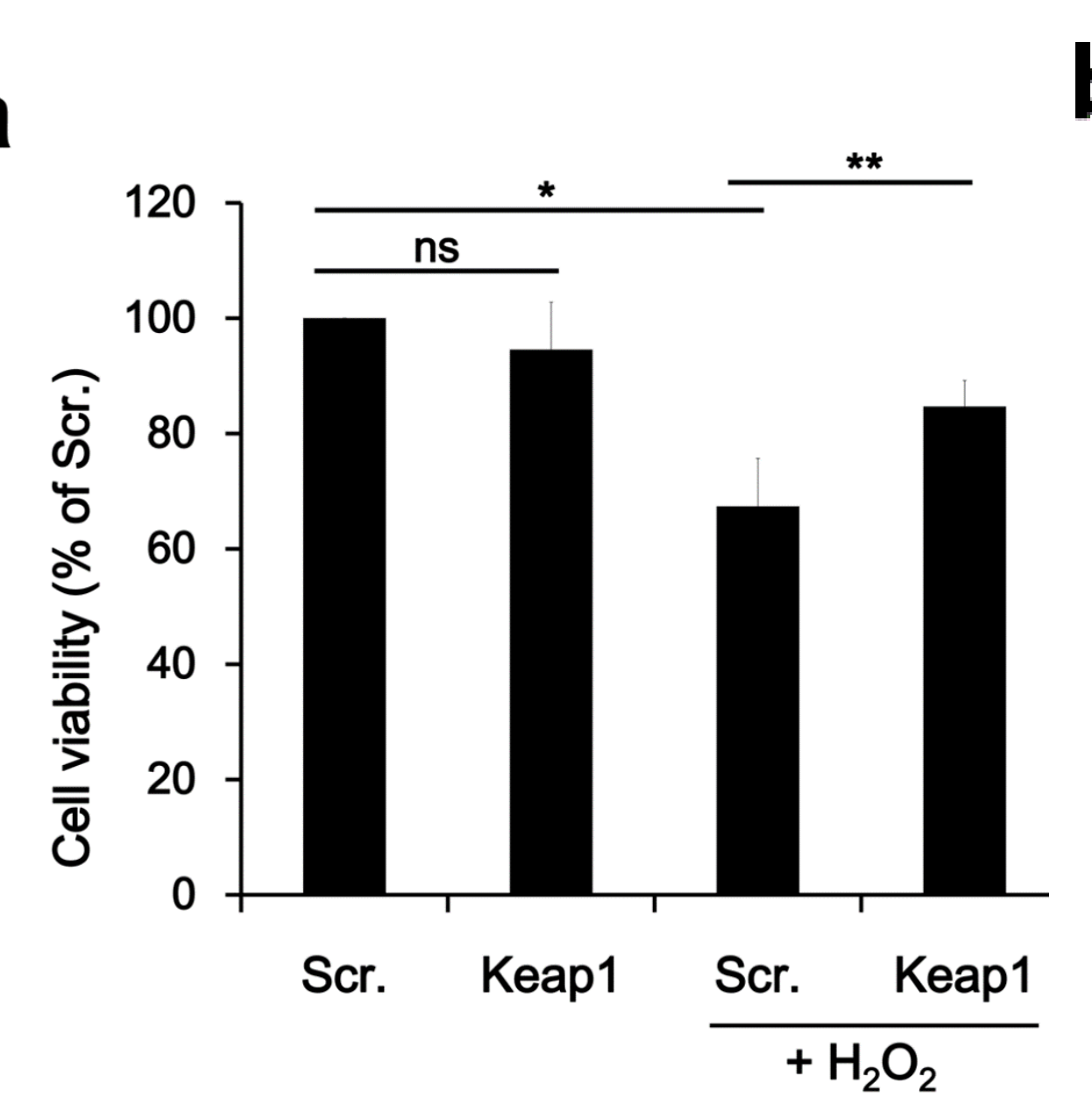

b $\begin{array}{r}\text { Scr. siRNA } \\ \text { Keap1 siRA } \\ \mathrm{H}_{2} \mathrm{O}_{2}(30 \mathrm{MM})\end{array}$

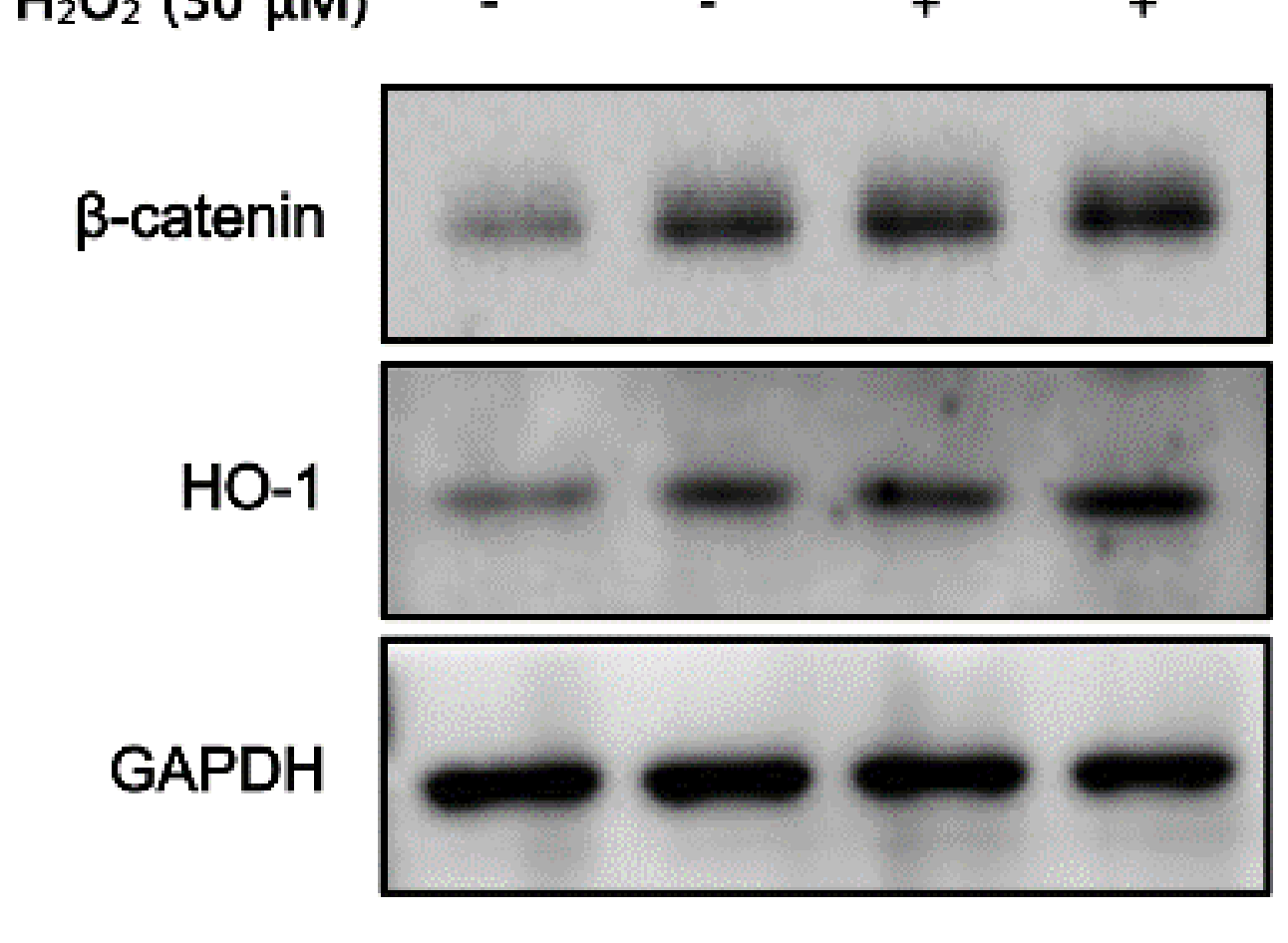
C $\begin{array}{r}\text { Scr. siRNA } \\ \text { Keap1 siRNA } \\ \text { HO-1 siRNA }\end{array}$

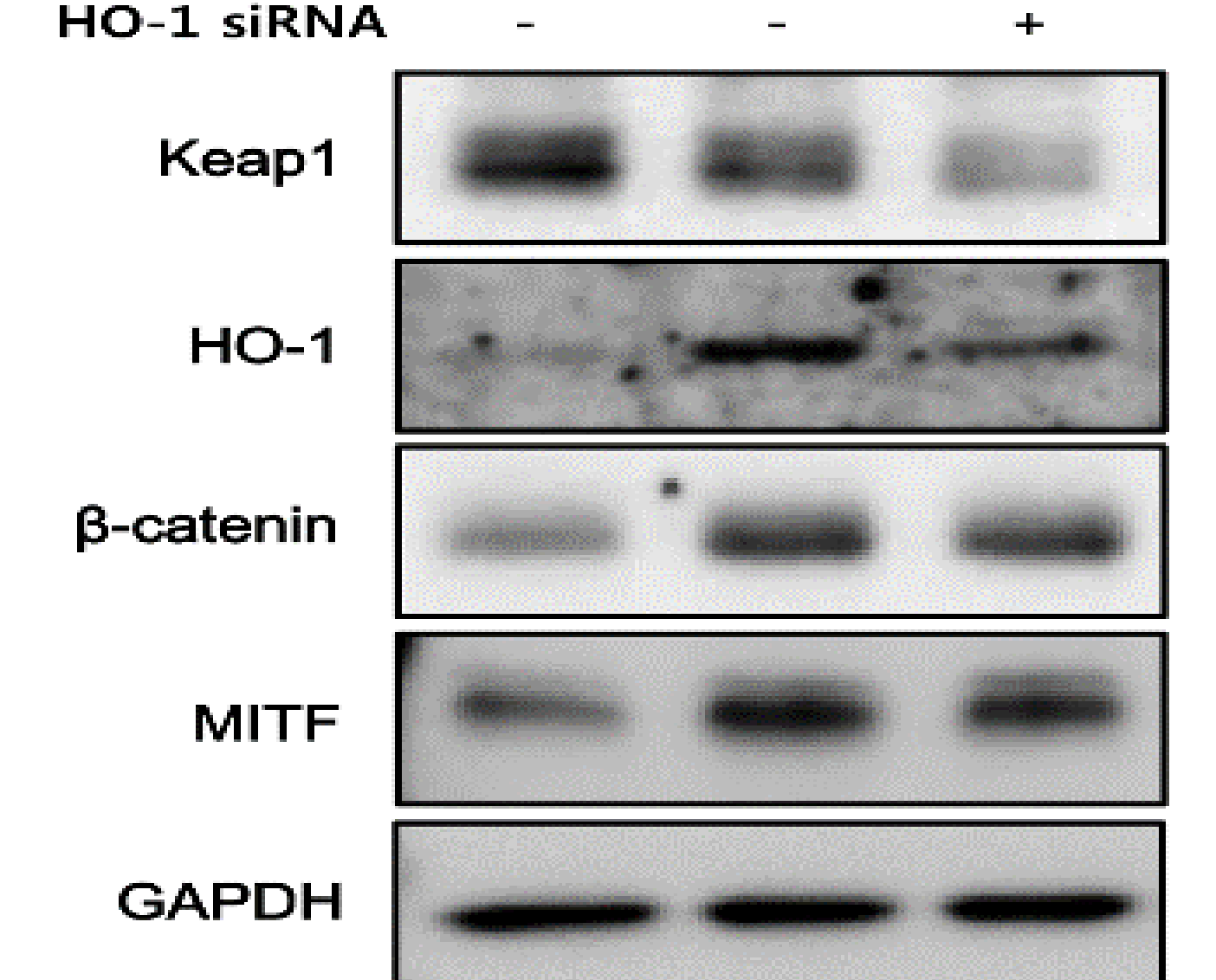

Fig. 6. Beta-catenin activation in Keap1 silenced PHEMs mediated by upregulation of HO-1

\section{Conclusion}

$>$ Keap1 silencing in melanocytes induced melanogenesis and the expression of melanogenesis-associated molecules through $\mathrm{HO}-1$-associated $\beta$-catenin activation. 